understanding of the risks associated with pulmonary misplacement and how to improve delivery of service is required. It is well known that feeding through a pulmonary placed NGT can lead to serious harm or mortality. The National Patient Safety Agency (NPSA) issued guidelines in 2011 to try to reduce reported cases of feeding into the lungs. However,it is unknown whether the introduction of an NG tube into the lungs, in itself can introduce infection.

Methods We conducted two snapshot audits at a tertiary centre between 2012-2013. Between these audits, organised training of nursing and core medical trainees was conducted. Audits involved spot-checks on 34 wards, assessing notes of all patients with NGTs in situ that day. These were then compared to NPSA guidelines.

We also did a retrospective study on the outcome of cases with pulmonary misplaced tubes, between 2012-2013. To identify these, chest X-ray reports were searched. Patient mortality and cases that later developed sepsis or chest infection were calculated. They were then compared to a group of controls that did have correctly placed NGTs.

Results The number of patients with NG tubes in situ were 38 and 46 in 2012 and 2013 respectively. None of these had cases of pulmonary misplacement. After the training that staff received there was a remarkable improvement in guideline adherence in 2013. Documentation of insertion increased by $46 \%$ ( $p<$ 0.001 ), and length of NGT recording increased by $53.9 \%$ ( $\mathrm{p}<$ $0.001)$. $\mathrm{pH}$ checks being the first line confirmation of position, rather than X-ray, increased by $33.8 \%(\mathrm{p}=0.002)$. In the 18 patients that had $\mathrm{pH}$ checks in 2013,7 patients did not require a second line Xray to check position.

From the radiology search there were 27 patients that did have pulmonary misplacement out of a total of 1332 (2.03\%). For mortality RR $=1.33(95 \%$ CI: $0.8424-2.1077, \mathrm{P}=$ 0.2199 ). For development of infection the $R R=1.94$ which was statistically significant (95\% CI: $1.1362-3.3207, \mathrm{P}=$ 0.0152). Direct logistic regression was performed on patient age to assess the impact on the likelihood of developing disease. However, this was not found to be statistically significant.

Conclusion It appears that training of both nursing and medical staff can have a huge benefit in increasing adherence to guidance. This study suggests that patients are nearly twice as likely to develop chest infection or sepsis after pulmonary misplacement of their NGT. Although other factors such as age have been shown to have little affect on this outcome, factors such as patient morbidity before misplacement could not be assessed. Disclosure of Interest None Declared.

\section{PTH-036 END OF LIFE CARE IN LIVER DISEASE}

F Chiplen*, S Lawton, G Linklater, S Salunke, L Cogdell, A Fraser, B Vijayan. NHS Grampian, Aberdeen, UK

\subsection{6/gutjnl-2014-307263.482}

Introduction Living and Dying Well: a national action plan for palliative and end of life care for Scotland calls for the identification, assessment, monitoring and care planning for palliative patients, irrespective of diagnosis or location. The primary aim of this project was to audit the assessment, care planning and recording of palliative status in the Gastroenterology Department in Aberdeen.

Methods Patients who died of chronic liver disease between July 2011 and May 2012 were identified using a retrospective notes review approach. A data capture sheet was devised in consultation with Senior Medical staff from the Gastroenterology and Palliative Medicine Departments.
Results Twenty patients were identified. The audit standard of $90 \%$ was met in the following: dying recognised and recorded in the medical notes; dying recognised and recorded in the nursing notes; DNACPR in place; record of discussion with the family and agreed plan with family; anticipatory prescribing. The $90 \%$ standard was not met for medication review, discontinuing unnecessary medication or non-essential monitoring/ interventions. None of the prognostic tools examined (MELD, Child Pugh, MI, GAHS) showed high sensitivity for recognising poor prognosis in this patient group. Almost all the patients who died in the acute sector had been admitted as unscheduled emergencies. None had any evidence of pre-admission advance care planning.

Conclusion This audit demonstrated a high standard of end-oflife care in the GI unit despite the difficulties identified in prognosticating in this patient group. There is a need to further explore whether advance care planning is possible and practicable for patients with chronic liver disease.

\section{REFERENCE}

Scottish Government Living and Dying Well: A National Audit Plan for Palliative and End-of-Life Care in Scotland The Scottish Government Edinburgh September 2008

Disclosure of Interest F. Chiplen: None Declared, S. Lawton: None Declared, G. Linklater: None Declared, S. Salunke Grant/ research support from: Shire Innovation award for registrars, L. Cogdell: None Declared, A. Fraser: None Declared, B. Vijayan: None Declared.

\section{PTH-037 ADHERENCE TO 2013 BSG BARRETT'S OESOPHAGUS SURVEILLANCE GUIDELINES COULD FACILITATE SAFE DISCHARGE OF PATIENTS}

${ }^{1} \mathrm{G}$ Walker*, 'N Moini, ${ }^{2} \mathrm{~F}$ Mayall, 'S Pugh. 'Gastroenterology, Musgrove Park Hospital, Taunton, UK; ${ }^{2}$ Pathology, Musgrove Park Hospital, Taunton, UK

\subsection{6/gutjnl-2014-307263.483}

Introduction The prevalence of Barrett's oesophagus (BO) is increasing and surveillance of this population is a growing burden for endoscopy units. ${ }^{1}$ Guidelines exist to facilitate quality surveillance but are poorly adhered to. ${ }^{2}$ We evaluated previous endoscopy (OGD) and histology reports of patients due to undergo BO surveillance in 2014 at a UK district general hospital. By using the 2013 BSG guidelines we decided if sufficient data were available to discharge patients or increase their surveillance interval. ${ }^{3}$

Methods The endoscopy department provided a list of all adult patients booked for BO surveillance in 2014. Electronic reports were used to find the length of $\mathrm{BO}$, presence/absence of intestinal metaplasia (IM), adherence to the Prague CandM criteria and compliance with the Seattle biopsy protocol (4 biopsies every $2 \mathrm{~cm}$ ). A Consultant pathologist clarified any uncertain histology reports. Outcomes after reviewing the cases were: 'discharged from surveillance', 'delayed OGD' or 'no change to date of OGD'. No patients with long segment $\mathrm{BO}(>3 \mathrm{~cm})$ had their endoscopies delayed. Finally hypothetical outcomes were calculated assuming all previous OGD's were compliant with BSG standards. Data were entered into an Excel spreadsheet and simple statistics used to analyse findings.

Results 125 patients were due BO surveillance in 2014. 96 male (77\%), average age 64 years (range 34-84). 76 (61\%) endoscopy reports were compliant with Prague CandM criteria. 33 (26\%) reports were compliant with the Seattle biopsy protocol, whilst $92(74 \%)$ were not. In $110(88 \%)$ cases no change was made to 